\title{
Wormlike chain or tense string? A question of resolution
}

\author{
J.-B. Fournier \\ Laboratoire de Physico-Chimie Théorique, ESPCI, 10 rue Vauquelin, F-75231 Paris cedex 05, \\ France
}

(Dated: November 21, 2018)

\begin{abstract}
It is shown that a wormlike chain, i.e., a filament with a fixed contour-length $\bar{S}$ and a bending elasticity $\kappa$, attached to a frame of length $L$, can be described — at low resolutions - by the same type of elastic free-energy as a tense string. The corresponding tension is calculated as a function of temperature, $L, \kappa$ and $\bar{S}$.
\end{abstract}

Elastic free-energies are used to describe the distorted states and fluctuations of softmatter Chaikin and Lubensky, 1995). The latter includes liquid-crystals (de Gennes and Prost, 1993), surfactant membranes or interfaces (Israelachvili, 1991), and polymers (Doi and Edwards. 1989). In this paper, we investigate how the elastic free-energy functional of a filament with a fixed contour-length and a bending rigidity (wormlike chain) depends on the spatial resolution at which it is described. We perform a finite renormalization group (RG) iteration in order to determine the free-energy associated with the length of the filament that is hidden at low-resolution. A similar study, focused on surfactant membranes, was recently published in a short paper (Fournier et al. 2001).

The filaments considered here are known as wormlike chains (Kratky and Porod, 1949), or semi-flexible polymers (Doi and Edwards, 1989; Landau and Lifshitz, 1971). At temperature T, a filament with a bending rigidity $\kappa$ has a persistence length $L_{p}=\kappa / k_{\mathrm{B}} T$ (Landau and Lifshitz, 1971): for contour-lengths $\bar{S} \ll L_{p}$ it resembles a flexible rod, while for $\bar{S} \gg L_{p}$ it resembles a wandering polymer. There are various natural realizations of semi-flexible polymers: actin cytoskeletal filaments, $L_{p} \simeq 4-17 \mu \mathrm{m}$ (Käs et al., 1994), microtubules, $L_{p} \simeq 6 \mathrm{~mm}$ (Gittes et al. 1993), and DNA, $L_{p} \simeq 50-350 \mathrm{~nm}$ (Hagerman, 1988; Marko and Siggia, 1995). Here we shall consider fluctuating filaments with $\bar{S} \gg L_{p}$, however they will be stretched by attachment to a

*Electronic address: jbf@turner.pct.espci.fr 
frame of width $L \lesssim \bar{S}$

The paper is organized as follows: in Sec. I we recall how a coarse-graining procedure allows to define different free-energies associated with different (spatial) resolutions of the same system; in Sec. II we describe the high-resolution free-energy of a wormlike chain; in Sec. III we calculate the corresponding low-resolution free-energy, and we show that it coincides with that of a tense string; in Sec. IV we summarize and discuss our results.

\section{CONSTRUCTING ELASTIC FREE-ENERGIES}

The free-energy of a system in thermodynamic equilibrium with a heath bath at temperature $T$ is defined by

$$
F[\mathcal{S}]=-k_{\mathrm{B}} T \ln \left(\sum_{i \in \mathcal{S}} e^{-\beta \mathcal{H}_{i}}\right),
$$

where $\beta=1 / k_{\mathrm{B}} T$ is the inverse temperature and $i$ labels the microscopic states (microstates) of the system, $\mathcal{H}_{i}$ being the energy, or Hamiltonian of state $i$. The above sum $\sum_{i \in \mathcal{S}} \exp \left(-\beta \mathcal{H}_{i}\right)$, also called restricted partition function $Z_{\mathcal{S}}$, runs over different subsets $\mathcal{S}$ of the phase space $\Gamma$ of all the microstates of the system. Each subset $\mathcal{S}$ defines a macrostate: it is a collection of microstates that we wish to consider macroscopically as a whole. $F[\mathcal{S}]$ is the free-energy of the macrostate $\mathcal{S}$; it is either a functional or a function depending on the explicit form of $\mathcal{S}$. For instance, in the standard thermodynamics of a fluid, the macrostates $\mathcal{S}$ are defined by specifying the number of particles $N$ and the volume $V$ of the fluid. Hence, $F$ is a function of the variables $T, V, N$.

It is most convenient to divide the phase space $\Gamma$ into a collection of disjoint macrostates $\mathcal{S}$. Then, since we know from statistical mechanics that the probability for the system to be in an arbitrary microstate $i$ is given by

$$
P_{i}=\frac{1}{Z_{\Gamma}} e^{-\beta \mathcal{H}_{i}}
$$

where $Z_{\Gamma}=\sum_{i \in \Gamma} \exp \left(-\beta \mathcal{H}_{i}\right)$, the quantity

$$
P[\mathcal{S}]=\frac{1}{Z_{\Gamma}} e^{-\beta F[\mathcal{S}]}
$$

is the probability to find the system in the macrostate $\mathcal{S}$. Indeed, from Eq. (1) we have $Z_{\Gamma}^{-1} \exp (-\beta F[\mathcal{S}])=\sum_{i \in \mathcal{S}} Z_{\Gamma}^{-1} \exp \left(-\beta \mathcal{H}_{i}\right)$. In other words, $F[\mathcal{S}]$ plays the role of a Hamiltonian for the macrostates $\mathcal{S}$. If the macrostates are well-chosen and large enough, the system will fluctuate little around the macrostate $\mathcal{S}^{\star}$ that minimizes $F[\mathcal{S}]$, i.e., around the most probable macrostate. The latter determines then the macroscopic "equilibrium state" of the system. 
In general, for a soft-matter system, one chooses for the macrostates $\mathcal{S}$ the profiles of the orderparameter field: the latter is defined as the local average of some interesting material property, e.g., for a nematic ${ }^{1}, Q_{i j}(\mathbf{r})=\left\langle m_{i}(\mathbf{x}) m_{j}(\mathbf{x})-\frac{1}{3} \delta_{i j}\right\rangle$, where $\mathbf{m}(\mathbf{x})$ is the orientation of the molecule situated at point $\mathbf{x}$, the average running over a small volume centered on $\mathbf{r}$. Note that the size $\Lambda_{0}^{-1}$ of this volume may be chosen at will and sets a cutoff $\Lambda_{0}$ for the order-parameter field. In the case of a filament, one may simply choose the positions $\mathbf{r}(s)$ of its constituting elements.

It is essential to note that we have a complete freedom to choose the "resolution" at which the phase space $\Gamma$ is coarse-grained into the macrostates $\mathcal{S}$. For example, on the one hand, we may be interested in the microscopic fluctuations of a bilayer membrane, as resolved at the scale of the membrane thickness $(\simeq 40 \AA)$. We would then define the free-energy $F[\mathcal{S}]$, as in Eq. (1), by summing over all the microstates $i$ compatible with a given shape $\mathcal{S}$ of the bilayer. Specifying one of these microstates would require specifying all the coordinates, velocities and conformations of all the lipids within the bilayer. On the other hand, we may be micro-manipulating a membrane under an optical microscope. In this case, we would only be interested in the Fourier components of the membrane shape with wavevector $q$ less than the typical wavevector of the visible light. We would then construct a free-energy functional $\bar{F}[\Sigma]$ by summing over all the microstates compatible with the poorly resolved shape $\Sigma$ of the membrane (Fournier et al., 2001). This would give us a tool more adapted to the experiment, since $\exp (-\beta \bar{F}[\Sigma])$ is directly the probability of observing $\Sigma$ (up to a normalization constant).

The free energy $\bar{F}[\Sigma]=-k_{\mathrm{B}} T \ln \sum_{i \in \Sigma} \exp \left(-\beta \mathcal{H}_{i}\right)$ associated with the coarse-grained states $\Sigma$ can be calculated also from

$$
\bar{F}[\Sigma]=-k_{\mathrm{B}} T \ln \left(\sum_{\mathcal{S} \in \Sigma} e^{-\beta F[\mathcal{S}]}\right)
$$

since

$$
\sum_{i \in \Sigma} e^{-\beta \mathcal{H}_{i}}=\sum_{\mathcal{S} \in \Sigma} \sum_{i \in \mathcal{S}} e^{-\beta \mathcal{H}_{i}}=\sum_{\mathcal{S} \in \Sigma} e^{-\beta F[\mathcal{S}]}
$$

Equations (田) and (5) define a "coarse-graining" procedure that can be carried-out for any partition $\Sigma$ of the phase space that is obtained by grouping the elements of a sub-partition $\mathcal{S}$. Note that the RG consists in applying this procedure repeatedly while rescaling the lengths and the fields at each step (Goldenfeld, 1992).

\footnotetext{
${ }^{1}$ a fluid with a long-range non-polar orientational order of its constituent molecules.
} 
It is usually impossible to directly perform the microscopic sum defining $\bar{F}[\Sigma]$. However, assuming on the grounds of symmetry ${ }^{2}$ a simple form for $F[\mathcal{S}]$, it may be possible to determine $\bar{F}[\Sigma]$ analytically from Eq. (雨). The functional $\bar{F}[\Sigma]$ will in general involve renormalized elastic constants (Chaikin and Lubensky, 1995) depending both on the elastic constants of $F[\mathcal{S}]$ and on temperature $T$ (these new elastic constants take into account the effects of the thermal fluctuation occurring at shorter scales). However, $\bar{F}[\Sigma]$ may have a functional form fairly different from that of $F[\mathcal{S}]$, as we shall see in the following.

It should be noted that this procedure, although akin to that of the RG, is different from the point of view of its scope. The scope of the RG is to identify the phases and critical points of a system by coarse-graining/rescaling infinitely. Here, the scope is to construct a free-energy functional adapted to the resolution at which the system is actually observed, or described (i.e., a tool fit to determine the shape and fluctuations of the system at this resolution).

\section{A WORMLIKE CHAIN AT HIGH RESOLUTION}

Let us consider a filament of fixed contour-length $\bar{S}$, attached at its extremities to two fixed points, separated by a distance $L$ along the $x$-axis (Fig. 1). We choose as macrostates $\mathcal{S}$ (see Sec. I) the microscopic shape of the filament, measured at the resolution that corresponds to its thickness. We restrict our attention to small deformations of the filament, hence we parameterize its shape by a normal displacement field $\mathbf{r}(x)$, with $\mathbf{r} \cdot \hat{\mathbf{x}}=0$ and $x \in[0, L]$. Each macrostate $\mathcal{S}$ thus corresponds to a field $\mathbf{r}(x)$. Let us introduce its Fourier transform $\mathbf{r}_{q}$ : extending $\mathbf{r}(x)$ periodically in such a way that $\mathbf{r}(x+L)=\mathbf{r}(x)$ for each $x$, we may write

$$
\begin{aligned}
\mathbf{r}(x) & =\frac{1}{\sqrt{L}} \sum_{q} \mathbf{r}_{q} e^{i q x} \\
\mathbf{r}_{q} & =\frac{1}{\sqrt{L}} \int_{0}^{L} d x \mathbf{r}(x) e^{-i q x}
\end{aligned}
$$

with $q=n \times 2 \pi / L$, where $n$ is a positive or negative integer. Since the highest resolution for $\mathbf{r}(x)$ corresponds to the filament's thickness $\simeq a$, we require

$$
|q|<\Lambda_{0} \equiv \frac{2 \pi}{a}
$$

The filament is assumed to be made of a fixed number of incompressible segments, hence its

\footnotetext{
${ }^{2}$ One usually represents the macrostates $\mathcal{S}$ by a small deformation field $\phi$, and one writes $F$ as the integral of a free-energy density expanded in power series of $\phi$ and its gradients.
} 


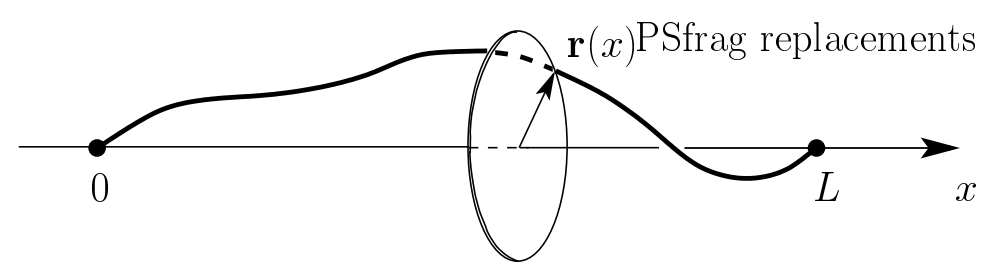

FIG. 1 Parameterization of the filament's shape. The disc indicates the plane normal to $\hat{\mathbf{x}}$, in which $\mathbf{r}(x)$ lives. In practice, we shall consider filaments that remain in the vicinity of the $x$-axis.

contour-length

$$
\bar{S}=\int_{0}^{L} d x \frac{d s}{d x} \simeq L+\int_{0}^{L} d x \frac{1}{2}\left(\frac{d \mathbf{r}}{d x}\right)^{2}
$$

is fixed. Here we have used the exact expression for the elementary arc-length $d s=$ $d x \sqrt{1+(d \mathbf{r} / d x)^{2}}$ and we have expanded the result up to second-order in $\mathbf{r}(x)$. In the following, assuming small tranverse excursions of the filament, we shall systematically expand all expressions in power-series of $\mathbf{r}(x)$, and retain only second-order terms.

Using Eq. (6) and $\int_{0}^{L} d x \exp (i q x)=L \delta_{q, 0}$, where $\delta$ is the Kronecker symbol, we have

$$
\bar{S}-L \simeq \sum_{|q|<\Lambda_{0}} \frac{1}{2} q^{2}\left|\mathbf{r}_{q}\right|^{2} .
$$

We have used the property $\mathbf{r}_{-q}=\mathbf{r}_{q}^{\star}$ that follows from the fact that $\mathbf{r}(x)$ is real-valued.

We assume that the free-energy of the filament, obtained in principle from the microscopic material properties through the procedure embodied in Eq. (11), is simply proportional to the integral of the square of the filament's curvature (Harris and Hearst, 1966; Kratky and Porod, 1949). More precisely,

$$
\begin{array}{ll}
F[\mathbf{r}]=\int_{0}^{\bar{S}} d s \frac{1}{2} \kappa\left(\frac{d \mathbf{t}}{d s}\right)^{2} & \text { if } \int_{0}^{L} d x \frac{d s}{d x}=\bar{S} \\
F[\mathbf{r}]=\infty & \text { otherwise. }
\end{array}
$$

Let us calculate the squared curvature up to second-order in $\mathbf{r}$. From $d \mathbf{M}=d x \hat{\mathbf{x}}+d \mathbf{r}$, where $\mathbf{M}$ is a vector the extremity of which runs along the filament, we deduce the tangent vector $\mathbf{t}=d \mathbf{M} / d s \simeq$ $\left[1-\frac{1}{2}(d \mathbf{r} / d x)^{2}\right] \hat{\mathbf{x}}+d \mathbf{r} / d x$. Hence, the curvature is $d \mathbf{t} / d s \simeq-\left[\left(d^{2} \mathbf{r} / d x^{2}\right) \cdot(d \mathbf{r} / d x)\right] \hat{\mathbf{x}}+d^{2} \mathbf{r} / d x^{2}$ and $(d \mathbf{t} / d s)^{2} \simeq\left(d^{2} \mathbf{r} / d x^{2}\right)^{2}$. The functional in Eq. (11) becomes, up to second order in $\mathbf{r}$,

$$
F[\mathbf{r}] \simeq \int_{0}^{L} d x \frac{1}{2} \kappa\left(\frac{d^{2} \mathbf{r}}{d x^{2}}\right)^{2}=\sum_{|q|<\Lambda_{0}} \frac{1}{2} \kappa q^{4}\left|\mathbf{r}_{q}\right|^{2}
$$




\section{THE SAME WORMLIKE CHAIN AT LOW RESOLUTION}

Let us now assume that we are not interested in the Fourier components of the filament's shape in the range $\Lambda<|q|<\Lambda_{0}$ (with $\Lambda>0$ ). For instance, we may be observing the filament with an experimental setup of resolution $2 \pi / \Lambda$. The coarse-grained macrostates $\Sigma$ of interest (see Sec. I) correspond then in real space to

$$
\mathbf{R}(\mathbf{x})=\sum_{|q|<\Lambda} \mathbf{r}_{q} e^{i q x}
$$

The field $\mathbf{R}(x)$, i.e., the poorly resolved shape of the wormlike chain, has a cutoff $\Lambda$ lower than that of $\mathbf{r}(x)$. We are interested in the free-energy $\bar{F}[\mathbf{R}]$ defined as in Eq. (幽 and not in $F[\mathbf{r}]$. Indeed, $\exp \{-\beta \bar{F}[\mathbf{R}]\}$ is, up to a multiplicative constant, the probability for the occurrence of a given low-resolution shape $\mathbf{R}(x)$-with any possible wrinkle in the either inaccessible or uninteresting range $\Lambda<|q|<\Lambda_{0}$; on the contrary, $\exp \{-\beta F[\mathbf{R}]\}$ would be the probability for the occurrence of a given low-resolution shape $\mathbf{R}(x)$ having no high-wavevector wrinkles at all (which is useless since it corresponds to a very particular and unlikely event). Note that in a purely harmonic systemwhich is not the case here because of the fixed contour-length constraint - the probability weight of the wrinkles is actually independent of the low-resolution shape $\mathbf{R}(x)$; in this case $\bar{F}[\mathbf{R}]=F[\mathbf{R}]$.

Following the procedure embodied in Eq. (四), we have

$$
\bar{F}[\mathbf{R}]=-k_{\mathrm{B}} T \ln \int \prod_{\Lambda<q<\Lambda_{0}} d \mathbf{r}_{q}^{\prime} d \mathbf{r}_{q}^{\prime \prime} \delta\left(L+\sum_{|q|<\Lambda_{0}} \frac{1}{2} q^{2}\left|\mathbf{r}_{q}\right|^{2}-\bar{S}\right) \exp \left(-\beta \sum_{|q|<\Lambda_{0}} \frac{1}{2} \kappa q^{4}\left|\mathbf{r}_{q}\right|^{2}\right) .
$$

Here, $\mathbf{r}_{q}^{\prime}$ and $\mathbf{r}_{q}^{\prime \prime}$ are the real and imaginary parts of $\mathbf{r}_{q}$, respectively. The integration variables [the wrinkles hidden in $\mathbf{R}(x)$ ] span the range $\Lambda<q<\Lambda_{0}$ of positive wavevectors only, because $\mathbf{r}_{-q}$ and $\mathbf{r}_{q}$ are not independent (they are complex conjugates). In principle, the above calculation is valid only for small values of $\mathbf{r}_{q}$, however, thanks to the Gaussian character of the integrand, we take the integrals as running over ] $-\infty, \infty[$. The Dirac delta-function takes into account the constraint on the filament's contour length [see Eq. (12)].

Let us call $S$ the contour-length of the low-resolution shape $\mathbf{R}(x)$, i.e., the apparent contourlength. The latter, which is no longer fixed, is given by

$$
S \simeq L+\sum_{|q|<\Lambda} \frac{1}{2} q^{2}\left|\mathbf{r}_{q}\right|^{2}
$$

Using this relation and the integral representation $\delta(x)=\int_{-\infty}^{\infty} d \lambda \exp (i \lambda x)$ of the delta-function, 
we may rewrite Eq. (15) in the form

$$
\begin{aligned}
\exp \{-\beta \bar{F}[\mathbf{R}]\}= & \int_{-\infty}^{\infty} \frac{d \lambda}{2 \pi} \int \prod_{\Lambda<q<\Lambda_{0}} d \mathbf{r}_{q}^{\prime} d \mathbf{r}_{q}^{\prime \prime} \times \\
& \exp \left[i \lambda\left(S-\bar{S}+\sum_{\Lambda<|q|<\Lambda_{0}} \frac{1}{2} q^{2}\left|\mathbf{r}_{q}\right|^{2}\right)-\beta \sum_{|q|<\Lambda_{0}} \frac{1}{2} \kappa q^{4}\left|\mathbf{r}_{q}\right|^{2}\right] .
\end{aligned}
$$

Since $\mathbf{r}_{q}=\mathbf{R}_{q}$ for $|q|<\Lambda$, we have

$$
\begin{aligned}
\exp \{-\beta \bar{F}[\mathbf{R}]\}= & \exp \left(-\beta \sum_{|q|<\Lambda} \frac{1}{2} \kappa q^{4}\left|\mathbf{R}_{q}\right|^{2}\right) \int_{-\infty}^{\infty} \frac{d \lambda}{2 \pi} e^{i \lambda(S-\bar{S})} \times \\
& \int \prod_{\Lambda<q<\Lambda_{0}} d \mathbf{r}_{q}^{\prime} d \mathbf{r}_{q}^{\prime \prime} \exp \left\{\frac{1}{2} \sum_{\Lambda<|q|<\Lambda_{0}}\left(i \lambda q^{2}-\beta \kappa q^{4}\right)\left[\left(\mathbf{r}_{q}^{\prime}\right)^{2}+\left(\mathbf{r}_{q}^{\prime \prime}\right)^{2}\right]\right\} .
\end{aligned}
$$

The rightmost integral, which is simply a product of separate Gaussian integrals, is equal to

$$
\left(\prod_{\Lambda<q<\Lambda_{0}} \sqrt{\frac{\pi}{\beta \kappa q^{4}-i \lambda q^{2}}}\right)^{4}=\exp \left[2 \sum_{\Lambda<q<\Lambda_{0}} \ln \left(\frac{\pi}{\beta \kappa q^{4}-i \lambda q^{2}}\right)\right] .
$$

For large values of $L$, we may replace the discrete sum by an integral. Hence Eq. (19) can be approximated by

$$
\exp \left[-\frac{L}{\pi} \int_{\Lambda}^{\Lambda_{0}} d q \ln \left(\beta \kappa q^{4}-i \lambda q^{2}\right)\right]
$$

up to a multiplicative constant independent of $\lambda$, which we discard as it merely shifts $\bar{F}[\mathbf{R}]$ by a fixed amount independent of $\mathbf{R}(x)$. We obtain

$$
\begin{aligned}
\exp \{-\beta \bar{F}[\mathbf{R}]\}= & \exp \left(-\beta \sum_{|q|<\Lambda} \frac{1}{2} \kappa q^{4}\left|\mathbf{R}_{q}\right|^{2}\right) \times \\
& \int_{-\infty}^{\infty} d \lambda \exp \left[i \lambda(S-\bar{S})-\frac{L}{\pi} \int_{\Lambda}^{\Lambda_{0}} d q \ln \left(\beta \kappa q^{4}-i \lambda q^{2}\right)\right]
\end{aligned}
$$

yielding

$$
\begin{aligned}
& \bar{F}[\mathbf{R}]=\int_{0}^{L} d x \frac{1}{2} \kappa\left(\frac{d^{2} \mathbf{R}}{d x^{2}}\right)^{2}+\phi(S) \\
& \phi(S)=-\frac{1}{\beta} \ln \int_{-\infty}^{\infty} d \lambda \exp \left[i \lambda(S-\bar{S})-\frac{L}{\pi} \int_{\Lambda}^{\Lambda_{0}} d q \ln \left(\beta \kappa q^{4}-i \lambda q^{2}\right)\right]
\end{aligned}
$$

Thus the free-energy associated to $\mathbf{R}(x)$ contains a bending term [as previously for $\mathrm{r}(x)$ ] plus a new potential $\phi(S)$ function of the contour-length of the low-resolution filament's shape. Note that corrections to $\kappa$ would have been generated if we had taken into account the non-harmonic terms 
in Eq. (13) (Helfrich, 1985; Peliti and Leibler, 1985). A correct treatment of these terms, including measure factors (Cai et al., 1994) is outside the scope of this paper.

In the thermodynamic limit $(L \rightarrow \infty)$, the integral in $\phi(S)$ can be approximated by its saddlepoint value (Walker, 1970). This yields

$$
\phi(S) \simeq-\frac{i \hat{\lambda}(S)}{\beta}(S-\bar{S})+\frac{L}{\pi \beta} \int_{\Lambda}^{\Lambda_{0}} d q \ln \left(\beta \kappa q^{4}-i \hat{\lambda}(S) q^{2}\right)
$$

with $\hat{\lambda}(S)$ the value of $\lambda$ that maximizes the integrand in Eq. (23); $\hat{\lambda}(S)$ is defined by

$$
\frac{\bar{S}-S}{L}=\frac{1}{\pi} \int_{\Lambda}^{\Lambda_{0}} \frac{d q}{\beta \kappa q^{2}-i \hat{\lambda}}
$$

Note that $\hat{\lambda}(S)$ actually lies on the imaginary axis of the complex plane. The Eqs. (24) and (25) define $\phi(S)$ implicitly.

A quantity of interest is

$$
\sigma(S)=\frac{d \phi(S)}{d S}
$$

It corresponds to the line tension associated with the low-resolution filament's shape $\mathbf{R}(x)$. Let us call $\tilde{\phi}(S, \hat{\lambda})$ the right-hand side of Eq. (24). Then we have

$$
\sigma(S)=\frac{d \tilde{\phi}(S, \hat{\lambda}(S))}{d S}=\frac{\partial \tilde{\phi}}{\partial S}+\frac{\partial \tilde{\phi}}{\partial \hat{\lambda}} \frac{d \hat{\lambda}}{d S}
$$

Since, by definition of $\hat{\lambda}, \partial \tilde{\phi} / \partial \hat{\lambda}=0$, we obtain

$$
\sigma(S)=-\frac{i \hat{\lambda}(S)}{\beta}
$$

Thus, the central result is the following: the line tension $\sigma(S)$, the primitive of which is the potential $\phi(S)$, is given by

$$
\pi \beta \kappa \frac{\bar{S}-S}{L}=\int_{\Lambda}^{\Lambda_{0}} \frac{d q}{q^{2}+\frac{\sigma(S)}{\kappa}}
$$

\section{A. Analysis}

Let us introduce the following dimensionless quantities:

$$
\begin{aligned}
s & =\pi \beta \kappa \Lambda_{0} \frac{\bar{S}-S}{L}, \\
\epsilon & =\frac{\Lambda}{\Lambda_{0}}, \\
\Sigma & =\frac{\sigma}{\kappa \Lambda_{0}^{2}} .
\end{aligned}
$$




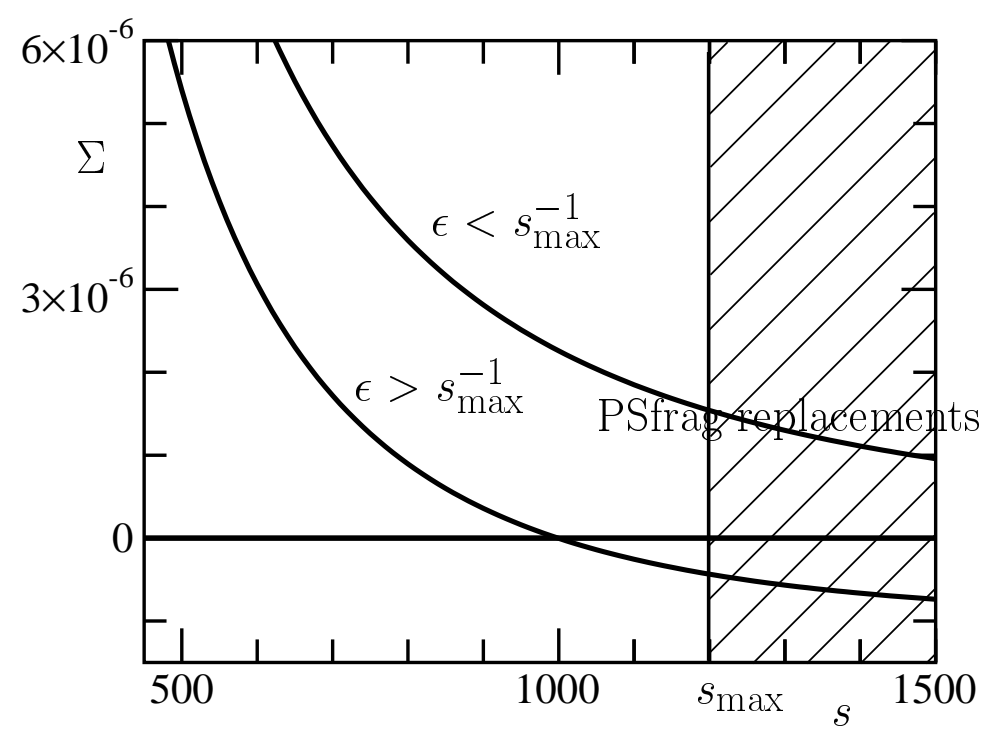

FIG. 2 Plot of the reduced effective wormlike chain's tension $\Sigma$ against the reduced hidden length $s$. The hatched region is forbidden. The top curve corresponds to $\epsilon=10^{-4}$ and the lower one to $\epsilon=10^{-3}$. For $\epsilon<s_{\max }^{-1}$ there is no way to obtain a vanishing tension: the filament behaves as a tense "string".

The parameter $s$ measures the hidden length of the filament. Since $L<S<\bar{S}$, we have

$$
0<s<s_{\max }, \quad \text { where } \quad s_{\max }=\pi \beta \kappa \Lambda_{0} \frac{\bar{S}-L}{L} .
$$

The dimensionless number $\beta \kappa \Lambda_{0}$, appearing in Eqs. (30) and (33), is the ratio of the persistence length of the filament, $L_{p}=\beta \kappa$, to its thickness $\approx \Lambda_{0}^{-1}$. For a wormlike chain, we expect

$$
\beta \kappa \Lambda_{0} \gg 1
$$

The parameter $\epsilon$ is the ratio of the high-resolution scale (the filament thickness) to the lowresolution scale $\Lambda^{-1}$. Obviously, we shall consider the case

$$
\epsilon \ll 1 \text {. }
$$

The parameter $\Sigma$ (having now a different meaning than in Sec. I) measures the filament's tension in units of $\kappa \Lambda_{0}^{2}$. Note that for $\beta \kappa \Lambda_{0} \gg 1$, the quantity $\kappa \Lambda_{0}^{2}$ is much larger than $k_{\mathrm{B}} T \Lambda_{0}$, which we would expect if the tension were of molecular origin.

In terms of these dimensionless quantities, the filament's tension, $\Sigma(s)$, is defined by the equation:

$$
s=\int_{\epsilon}^{1} \frac{d k}{k^{2}+\Sigma}
$$


Let us now study the various regimes displayed by $\Sigma(s)$. For $s \rightarrow 0, \Sigma$ diverges as $(1-\epsilon) / s$, as can be easily seen by neglecting $k^{2}$ with respect to $\Sigma$ in Eq. (36). $\Sigma$ decreases monotonically and vanishes at $s=s^{\star}(\epsilon)$ with $($ for $\epsilon \ll 1)$

$$
s^{\star} \simeq \frac{1}{\epsilon}
$$

it becomes then negative. When $\epsilon<s_{\max }^{-1}, s^{\star}$ lies beyond $s_{\max }$ and is thus unreachable (Fig. 2). Hence, as in the membrane case discussed by Fournier et al. (2001), there are two regimes: for $\epsilon>s_{\max }^{-1}$, i.e., when the resolution $\Lambda$ is higher than $\Lambda_{0} / s_{\max }$, the filament is in a floppy regime, while for $\epsilon<s_{\max }^{-1}$ (low resolution), it is in a tense regime.

In the floppy regime, the low-resolution contour $\mathbf{R}(x)$ exhibits a loose shape with an apparent contour-length fluctuating in the vicinity of the value $S^{\star}$ (associated to $s^{\star}$ ) that minimizes the potential $\phi(S)$. At $S=S^{\star}$, we have $\sigma=0$ : the tension effectively vanishes. This is reminescent of the case of membranes (David and Leibler, 1991; Seifert, 1995).

\section{B. Effectively a tense string}

In the tense regime, the apparent contour-length minimizing $\phi(S)$ corresponds to the lowest possible value of $\Sigma$, attained at $s=s_{\max }$, which corresponds to the lowest possible value of $S$, i.e., L. The low-resolution, apparent shape of the filament is thus the straight line joining the two fixed extremities (Fig. 3). The filament, described at such low resolutions, behaves therefore effectively as a tense string of tension $\sigma_{0}=\sigma(L)$. As one pulls on the "string", the apparent contour-length increases $(S>L)$ and so does the tension.

Although Eq. (36) cannot be solved analytically for $\Sigma$, approximate solutions can be obtained in the tense regime both for medium and high tensions. Assuming $\epsilon \ll 1$, we replace the lowest bound of the integral in Eq. (36) by zero. This gives $s \simeq(1 / \sqrt{\Sigma}) \arctan (1 / \sqrt{\Sigma})$, which yields the following approximations: $s \simeq \pi /(2 \sqrt{\Sigma})$ for $0<\Sigma \ll 1$ (medium tensions), and $s \simeq 1 / \Sigma$ for $\Sigma \gg 1$ (high tensions).

For real wormlike chains and in the high tension case, we should actually take into account the finite stretching elasticity of the filament: this would modify our results. We shall therefore concentrate on the medium tension states of the tense regime. The relation $s \simeq \pi /(2 \sqrt{\Sigma})$ yields

$$
\sigma \simeq \frac{1}{4 \beta^{2} \kappa}\left(\frac{L}{\bar{S}-S}\right)^{2}
$$

This represents the free-energy we have to pay in order to increase the apparent contour-length of the filament by a unit amount. It is worth noticing the intrinsic character of this expression: it 


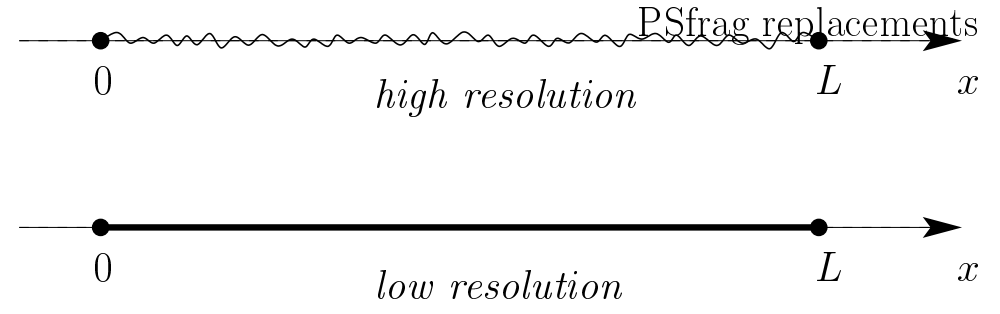

FIG. 3 The same wormlike chain viewed at high and low resolutions. The elasticity describing the lowresolution shape and its fluctuations (not drawn) is similar to that of a tense string.

is independent both of the microscopic cutoff $\Lambda_{0}$ and of the resolution $\Lambda$ at which the filament is observed. For Eq. (38) to hold, it is necessary that

$$
\Lambda \ll \Lambda_{c}=\frac{1}{\pi \beta \kappa} \frac{L}{\bar{S}-L}
$$

in order to be well into the tense regime $\left(\epsilon \ll s_{\max }^{-1}\right)$.

\section{CONCLUSION}

We have shown that at spatial resolutions $\Lambda$ much less than the wavevector $\Lambda_{c}$ defined above, a wormlike chain, i.e., a filament with a bending elasticity $\kappa$ and a fixed contour-length $\bar{S}$, behaves as a tense string when it is attached at its extremities to a frame of length $L$. In other words, if one forgets about the microscopic wrinkles of the filament by considering only its low-resolution shape $\mathbf{R}(x)$ (the Fourier transform of the microscopic shape truncated at the cutoff $\Lambda$ ), one is actually considering a system that has the same type of elastic free-energy as a tense string. Indeed, in the minimum energy configuration, the low-resolution shape of the filament is straight and its tension is given by

$$
\sigma_{0} \simeq \frac{1}{4 \beta^{2} \kappa}\left(\frac{L}{\bar{S}-L}\right)^{2},
$$

which follows from Eq. (38) by setting $S=L$. Again, we note that this effective tension is independent of the resolution $\Lambda$ (as long as $\Lambda \ll \Lambda_{c}$ ). For small deformations about this straight state, one can neglect the fact that $\sigma$ increases with $S$ as one pulls on the "string". Then, the elastic free-energy of the filament, as seen at low-resolution, is

$$
\bar{F}[\mathbf{R}] \simeq \int_{0}^{L} d x \frac{1}{2} \sigma_{0}\left(\frac{d \mathbf{R}}{d x}\right)^{2} .
$$

In this last expression, we have neglected the remaining energy associated with the bending of the filament [see Eq. (22)]. It is actually negligible in the tense regime: for all the permitted 
wavevectors $(q<\Lambda)$, we have $q \ll \Lambda_{c}$, and therefore $\sigma_{0} q^{2} \gg \kappa q^{4}$ since $\sqrt{\sigma_{0} / \kappa} \approx \Lambda_{c}$, as can be seen from Eqs. (39) and (40).

Although this paper is focused on the concept of effective tension for a wormlike chain viewed at a low resolution, Eqs. (22), (23) and (29) actually give the effective free-energy fit to describe an attached wormlike chain viewed at any resolution.

It is interesting to compare the present results with those of previous works. For instance, using the wormlike chain model, Marko and Siggia (1995) have calculated the force $f$ needed to extend a DNA molecule attached at its extremities. In agreement with experiments (Smith et al., 1992), they found that the end-to-end distance approaches the DNA contour-length as $f^{-1 / 2}$. Precisely, they obtained $\left(4 f L_{p} / k_{\mathrm{B}} T\right)^{-1 / 2} \simeq 1-L / \bar{S}$ in the strong force limit. Since $\beta \kappa \equiv L_{p}$, Eq. (40) reproduces exactly the same result, upon identifying the effective tension $\sigma_{0}$ with the force $f$.

Since the early work of Helfrich (Helfrich, 1985; ; Peliti and Leibler, 1985), a large literature has been devoted to the renormalization of the elastic constants of membranes. No similar work has been performed on wormlike chains, however: this is in part because it is possible to directly calculate the tangent-tangent correlation function by analogy with the diffusion on a unit sphere (Harris and Hearst, 1966). A large amount of work has been devoted instead to the interpolation between the wormlike and the Gaussian chain (Aragón and Pecora, 1985; Bawendi and Freed, 1985; Ha and Thirumalai, 1995). To the best of my knowledge, however, there has been no previous analysis in the spirit of the present paper.

\section{Acknowledgments}

I wish to thank A. Ajdari, T. B. Liverpool, T. Lubensky and L. Peliti for useful discussions.

\section{References}

Aragón, S. R., and R. Pecora, 1985, Macromolecules 18, 1868.

Bawendi, M. G., and K. F. Freed, 1985, J. Chem. Phys. 83, 2491.

Cai, W., T. C. Lubensky, P. Nelson, and T. Powers, 1994, J. Phys. II France 4, 931.

Chaikin, P. M., and T. C. Lubensky, 1995, Principles of Condensed Matter Physics (Cambridge University

Press, Cambridge).

David, F., and S. Leibler, 1991, J. Phys. II France 1, 959.

Doi, M., and S. F. Edwards, 1989, The Theory of Polymer Dynamics (Clarendon Press, Oxford).

Fournier, J.-B., A. Ajdari, and L. Peliti, 2001, Phys. Rev. Lett. 86, 4970.

de Gennes, P.-G., and J. Prost, 1993, The Physics of Liquid Crystals (Clarendon, Oxford). 
Gittes, F., B. Mickey, J. Nettleton, and J. Howard, 1993, J. Cell. Biol. 120, 923.

Goldenfeld, N., 1992, Lectures on Phase Transitions and the Renormalization Group (Frontiers in PhysicsPerseus Books, Reading, Massachusetts).

Ha, B.-Y., and D. Thirumalai, 1995, J. Chem. Phys. 103, 9408.

Hagerman, P. J., 1988, Annu. Rev. Biophys. Biophys. Chem. 17, 265.

Harris, R. A., and J. E. Hearst, 1966, J. Chem. Phys. 44, 2595.

Helfrich, W., 1985, J. Physique 46, 1263.

Israelachvili, J., 1991, Intermolecular $\& 3$ Surface Forces (Academic Press, New York).

Käs, J., H. Strey, and E. Sackmann, 1994, Nature 368, 226.

Kratky, O., and G. Porod, 1949, Recl. Trav. Chim. Pays-Bas 68, 1106.

Landau, L. D., and E. M. Lifshitz, 1971, Statistical Physics (Pergamon, Oxford).

Marko, J. F., and E. D. Siggia, 1995, Macromolecules 28, 8759.

Peliti, L., and S. Leibler, 1985, Phys. Rev. Lett. 54, 1690.

Seifert, U., 1995, Z. Phys. B 97, 299.

Smith, S. B., L. Finzi, and C. Bustamante, 1992, Science 258, 1122.

Walker, M., 1970, Mathematical Methods of Physics (Addison-Wesley, New-York). 